\title{
Endoscopic transduodenal cholecystolithotomy and cholecystolithotripsy
}
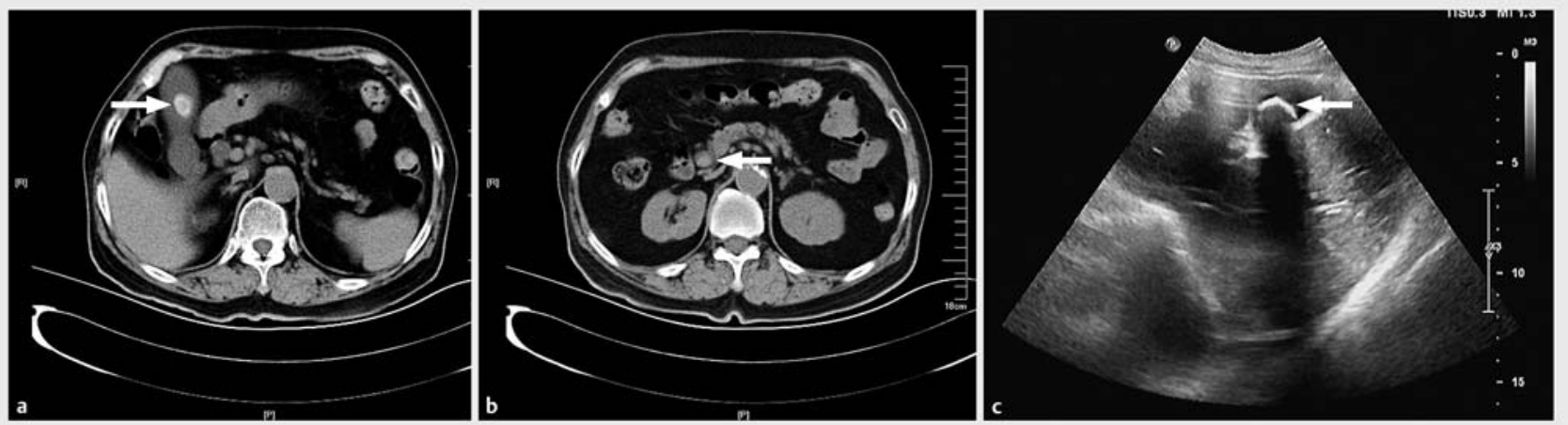

- Fig. 1 Imaging prior to treatment. Computed tomography scan revealed a gallbladder stone (a, arrow) and common bile duct stones (b, arrow). c Transabdominal ultrasound scan showed the gallbladder stone (arrow).

Laparoscopic cholecystectomy is generally accepted as the gold standard treatment for symptomatic gallstones [1,2]. For patients who are not suitable operative candidates, endoscopic ultrasound (EUS)-guided cholecystoduodenostomy or cholecystogastrostomy can be performed using a double-flanged, fully covered, metal stent as a bridge to further transduodenal or transgastric cholecystolithotomy $[3,4]$. Given that the diameter of the stent is limited, it was previously believed that only gallstones of less than $1 \mathrm{~cm}$ in diameter should be removed by this novel technique. Here, we report a patient with a symptomatic gallstone larger than $1 \mathrm{~cm}$ who successfully underwent endoscopic transduodenal cholecystolithotripsy via a double-flanged, fully covered, metal stent.

An 80-year-old woman was admitted to the medical center with chills, fever, and nausea for 17 days, as well as jaundice for 7 days. An initial contrast computed tomography (CT) scan and transabdominal ultrasound revealed gallbladder and common bile duct (CBD) stones ( $\triangleright$ Fig.1). Further EUS demonstrated that the stone in the gallbladder was approximately $20 \times 16 \mathrm{~mm}$ in size, and the stones in the CBD were $4-7 \mathrm{~mm}$ in diameter ( $\mathbf{F i g} \mathbf{2} \mathbf{a}, \mathbf{b}$ ). As the poor medical condition of this patient made her an unsuitable operative candidate, endoscopic sphincterectomy and EUS-guided cholecystolithotomy were planned to treat the cholelithiasis and choledocholithiasis.

After informed consent had been obtained, the EUS-guided cholecystoduodenostomy was carried out using a previously reported technique $[4,5]$. A longitudinal echoendoscope with a working channel of $3.8 \mathrm{~mm}$ was introduced into the duodenum to scan for and mark the puncture point. An EchoTip Ultra EUS needle (22 G; Cook Medical Inc., Bloomington, Indiana, USA) was introduced

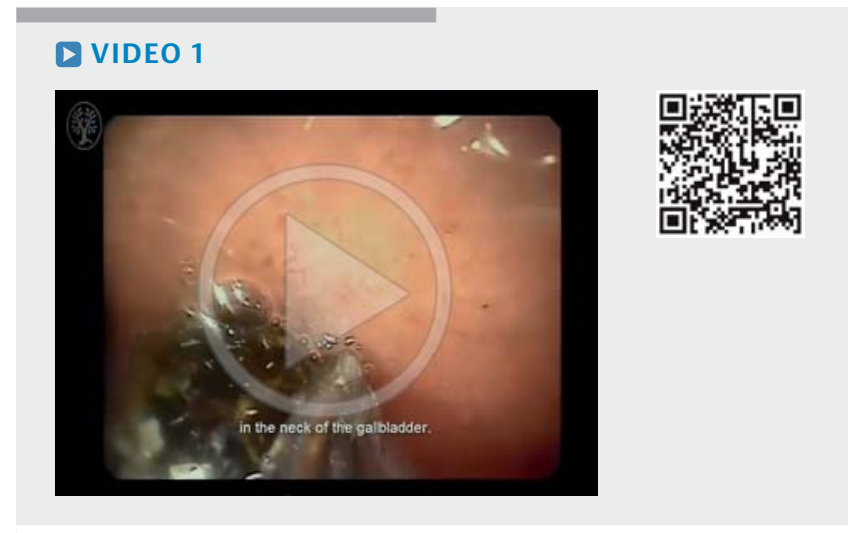

- Video 1: Endoscopic transduodenal cholecystolithotomy and cholecystolithotripsy. via the working channel of the echoendoscope, and the gallbladder was punctured under EUS guidance. Bile was aspirated, and $10 \mathrm{~mL}$ of radiographic contrast was injected to confirm that the punctured structure was the gallbladder. Under the guidance of EUS and fluoroscopy, the double-flanged stent $(2.5 \mathrm{~cm}$ in length, $1.5 \mathrm{~cm}$ in width, $1.2 \mathrm{~cm}$ in diameter) was placed and deployed as a bridge between the gallbladder and the duodenum. Next, endoscopic sphincterectomy was performed, which successfully removed the stones from the CBD. 

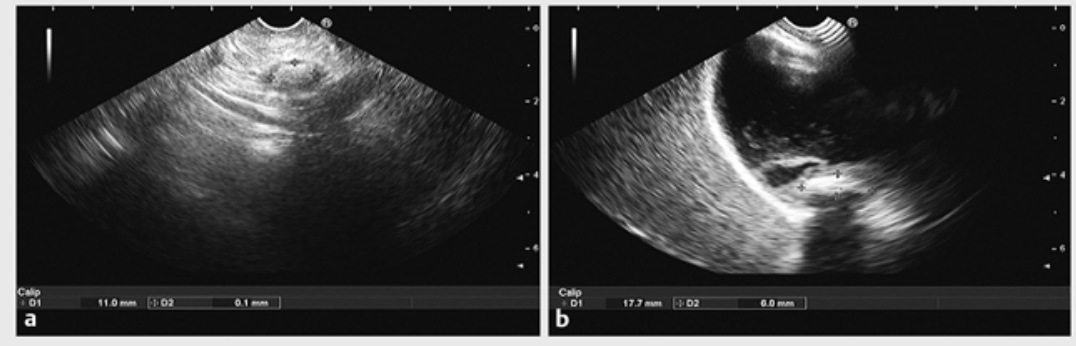

- Fig. 2 Endoscopic ultrasound images prior to treatment. a The stone in the gallbladder was approximately $20 \times 16 \mathrm{~mm}$ in size. $\mathbf{b}$ The stones in the common bile duct were $4-7 \mathrm{~mm}$ in diameter.
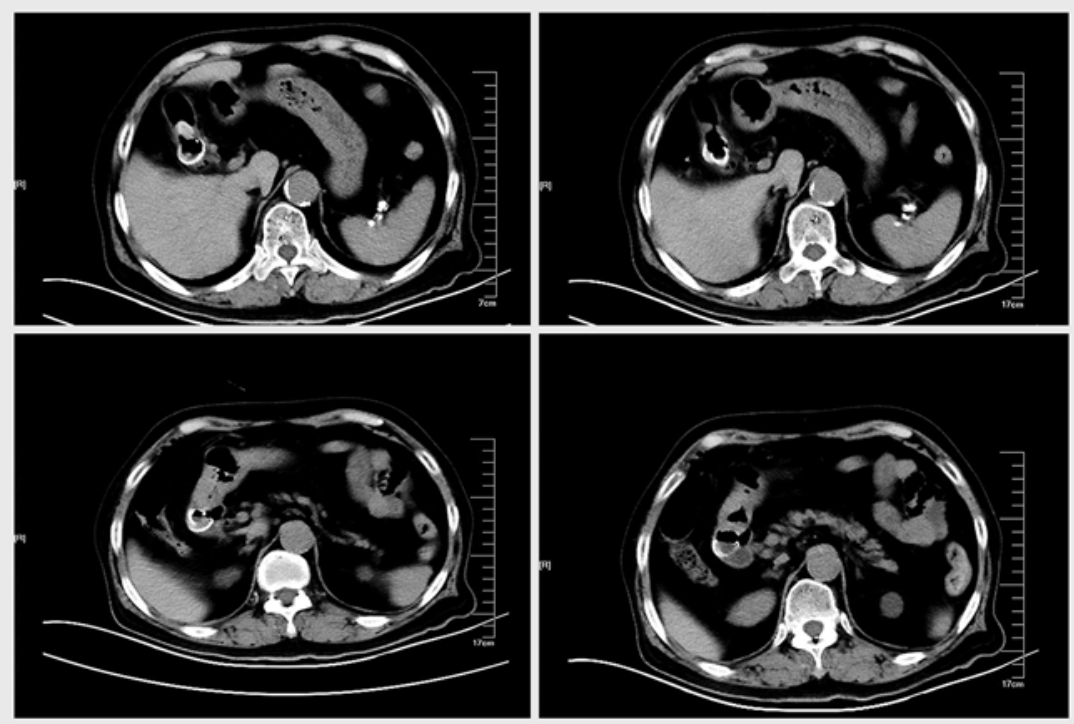

- Fig. 3 Computed tomography was performed to confirm the formation of a fistula between the gallbladder and duodenum, and to ensure that the stent remained in place.

No symptoms of peritonitis were observed during the postoperative period, and a normal diet was resumed 48 hours after the operation ( $\vee$ Video 1 ).

Two weeks after the cholecystoduodenostomy procedure with stent placement, the patient underwent transduodenal cholecystolithotomy. Prior to this operation, CT was performed to confirm the formation of a fistula between the gallbladder and duodenum, and to ensure that the stent remained in place ( $\triangleright$ Fig.3). A gastrointestinal endoscope with a working channel of $2.8 \mathrm{~mm}$ was advanced into the gallbladder via the stent. A stone measuring approximately $20 \times 16 \mathrm{~mm}$ was observed in the neck of the gallbladder. It could not be extricat- ed by a stone basket through the metal stent, leaving cholecystolithotripsy as the only way to definitively treat this condition.

First, a stone basket was inserted into the gallbladder via the working channel to catch the stone. The basket with stone was then withdrawn from the gallbladder and locked within the flared cholecystic end of the stent. Next, the basket was cut off and the lithoclast was applied to break the stone into two pieces, which could then be discharged from the gallbladder via the stent. The stent was then removed. The inside of the gallbladder was carefully cleaned. Finally, radiography via balloon catheter was performed, which showed no residual stones. An endoscopic nasobiliary drain (ENBD) was placed in the gallbladder.

Postoperatively, the patient had no complaints, and no abnormal laboratory results were found. Radiography performed 24 hours later via the ENBD revealed a large amount of contrast medium leaking from the gallbladder into the duodenum, indicating that the fistula was unhealed. A repeat radiographic study 48 hours later showed a decrease in leakage and the ENBD was subsequently removed. Esophagogastroduodenoscopy performed with transparent cap 60 hours later revealed a closed fistula and an ulcer was seen ( $\triangleright$ Video 1 ). After 5 days of antibiotics and proton pump inhibitor treatment, the patient was discharged without complications.

To the authors' knowledge, this is the first EUS-guided cholecystoduodenostomy plus endoscopic transduodenal cholecystolithotripsy reported in the world. Previous studies have indicated that gallstones smaller than $1 \mathrm{~cm}$ can be removed via a fistula formed by a stent between the gallbladder and the stomach or duodenum [3-6]. Here, we described a new technique to treat gallstones larger than $1 \mathrm{~cm}$. We strongly recommend that cholecystolithotripsy should be performed within the stent to protect the gallbladder and duodenum. The success of this endoscopic transduodenal cholecystolithotripsy method indicates that the size of the gallstone is no longer a limiting factor in popularizing this new technology [7].

Endoscopy_UCTN_Code_TTT_1AS_2AD

Competing interests

None

The Authors

Guoxin Wang, Xiang Liu, Sheng Wang, Nan Ge, Jintao Guo, Siyu Sun

Endoscopy Center, Shengjing Hospital of China Medical University, Shenyang, China 
Siyu Sun, MD, PhD

Endoscopy Center, Shengjing Hospital of China Medical University, No. 36 Sanhao Street, Heping District, Shenyang 110004 , Liaoning Province, China sun-siyu@163.com

\section{Acknowledgment}

This study was supported by the National Natural Science Foundation of China (Grant No. 81470908).

\section{References}

[1] Fisichella PM, DeMeester SR, Hungness E et al. Emerging techniques in minimally invasive surgery .Pros and cons. J Gastrointest Surg 2015; 19: 1355 - 1362

[2] Netinatsunton N, Attasaranya S, Sottisuporn J et al. Comparing cost-effectiveness between endoscopic ultrasound and endoscopic retrograde cholangiopancreatography in diagnosis of common bile duct stone in patients with predefined risks: a study from a developing country. Endosc Ultrasound 2016; 5: 165 - 172

[3] Ge N, Wang Z, Sun S et al. EUS assisted transmural cholecystogastrostomy fistula creation as a bridge for endoscopic internal gallbladder therapy using a novel fully covered metal stent. BMC Gastroenterol 2014; 14: 164

[4] Ge N, Sun S, Sun S et al. Endoscopic ultrasound-assisted transmural cholecystoduodenostomy or cholecystogastrostomy as a bridge for per-oral cholecystoscopy therapy using double-flanged fully covered metal stent. BMC Gastroenterol 2016; 16: 9

[5] Ge N, Wang S, Wang S et al. Endoscopic ultrasound-assisted cholecystogastrostomy by a novel fully covered metal stent for the treatment of gallbladder stones. Endosc Ultrasound 2015; 4: 152-155

[6] Mukai S, Itoi T. How should we use endoscopic ultrasonography-guided biliary drainage techniques separately? Endosc Ultrasound 2016; 5: 65-68

[7] Satyarth CH, Sun S. Endoscopic ultrasoundguided gallbladder drainage: redefines the boundaries. Endosc Ultrasound 2016; 5: $281-283$

\section{Bibliography}

DOI http://dx.doi.org/10.1055/s-0043-102394

Endoscopy 2017; 49: E111-E113

(c) Georg Thieme Verlag KG

Stuttgart · New York

ISSN 0013-726X 